\title{
Estimating Vehicle Stability Region Based on Energy Function
}

\author{
Yu-guang Yan, ${ }^{1,2}$ Hong-guo $\mathrm{Xu},{ }^{1}$ and Hong-fei Liu ${ }^{1}$ \\ ${ }^{1}$ College of Transportation, Jilin University, Changchun 130025, China \\ ${ }^{2}$ College of Computer, Changchun Normal University, Changchun 130032, China
}

Correspondence should be addressed to Yu-guang Yan; 16627147@qq.com

Received 17 July 2014; Accepted 13 September 2014

Academic Editor: Yongjun Shen

Copyright (C) 2015 Yu-guang Yan et al. This is an open access article distributed under the Creative Commons Attribution License, which permits unrestricted use, distribution, and reproduction in any medium, provided the original work is properly cited.

\begin{abstract}
In order to improve the deficiency of vehicle stability region, according to vehicle nonlinear dynamic model, method of estimating vehicle spatial stability region was proposed. With Pacejka magic formula tire model, nonlinear 3DOF vehicle model was deduced and verified though vehicle test. Detailed detecting system and data processing were introduced. In addition, stability of the vehicle system was discussed using Hurwitz criterion. By establishing energy function for vehicle system, the vehicle's stability region in $20 \mathrm{~m} / \mathrm{s}$ was estimated based on Lyapunov theorem and vehicle system characteristics. Vehicle test in the same condition shows that the calculated stability region defined by Lyapunov and system stability theorem has good effect on characterized vehicle stability and it could be a valuable reference for vehicle stability evaluation.
\end{abstract}

\section{Introduction}

It is clear that stability region for vehicle stability evaluation has been extensively studied in our country and abroad now. Up to date, some researchers have analyzed stability region applying simple vehicle model [1, 2]. Typically, in the 1990s, Inagaki, Samsundar, Yasui, and Stotsky proposed analytical expression for vehicle plane stability region based on simplified cubic tire model and deduced plane oval stability region by simulation [3-6]. In 2002, Ko and Lee estimated plane stability region of vehicle by side slip angle and yaw rate according to topology theory and 2DOF vehicle model [7]. Similarly, in 2007, Shen et al. estimated singular points of vehicle and studied stability characteristics of vehicle [8].

Many reference classical conclusions play a positive role for vehicle stability region; however there are still some limitations: first, most of the traditional studies were based on simplified 2DOF vehicle models, so vehicle state could not be comprehensively considered. In addition, it is crystal clear that traditional vehicle dynamic model with simplified tire formula, such as linear tire model, was conveniently calculated but it still had great deviation compared with fact, and as a result, the accuracy of vehicle region was unsatisfied. Furthermore, traditional researches for vehicle region boundary conditions had insufficient basis, which affect the accuracy as well.
Therefore, an improved nonlinear vehicle dynamic model with Pacejka magic formula is introduced, and the stability region of the vehicle is estimated based on energy method. It is valuable for evaluating the stability of vehicle and improving traffic safety.

\section{Vehicle Nonlinear Dynamic Model}

2.1. Vehicle Differential Equations. Figures 1(a) and 1(b) illustrate the 3DOF vehicle model or double-axis vehicle and coordinate systems. Suppose the vehicle is steering at a speed without braking and driving forces, pitch movement, and longitudinal tire force and align toque could be ignored in this condition. So the established vehicle 3DOF model consists of lateral motion, yaw, and roll motions.

Corresponding equations of the vehicle model are described as follows:

$$
\begin{gathered}
m\left(\dot{v}_{y}+v_{x} \omega\right)-m_{s} h_{s} \ddot{\psi}_{s}=F_{y f}+F_{y r}, \\
I_{z} \dot{\omega}=a F_{y f}-b F_{y r}, \\
I_{x s} \ddot{\psi}_{s}+m_{s} h_{s}\left(\dot{v}_{y}+v_{x} \omega-h_{s} \ddot{\psi}_{s}\right)=m_{s} g h_{s} \psi_{s}-M_{\psi},
\end{gathered}
$$




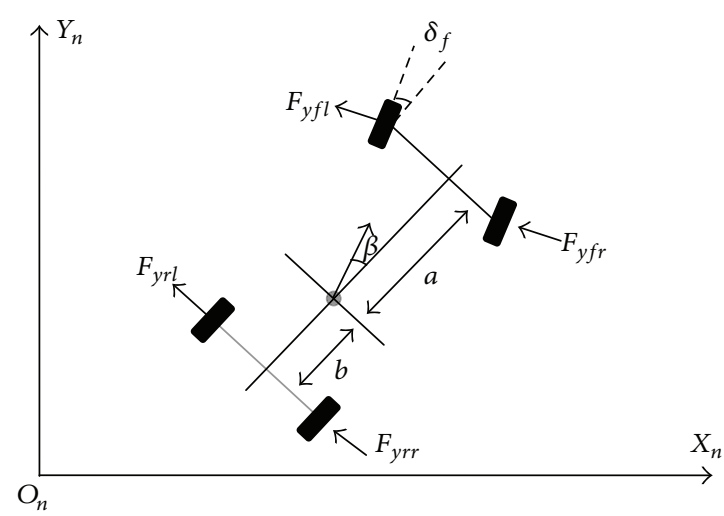

(a) Plane movement

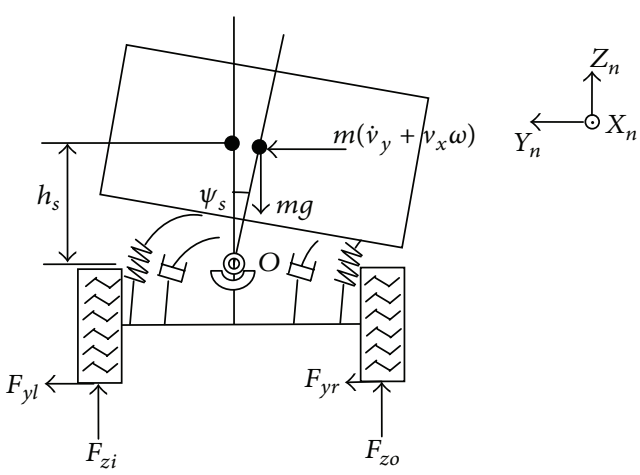

(b) Roll movement

FIgURE 1: Schematic diagram of body movement.
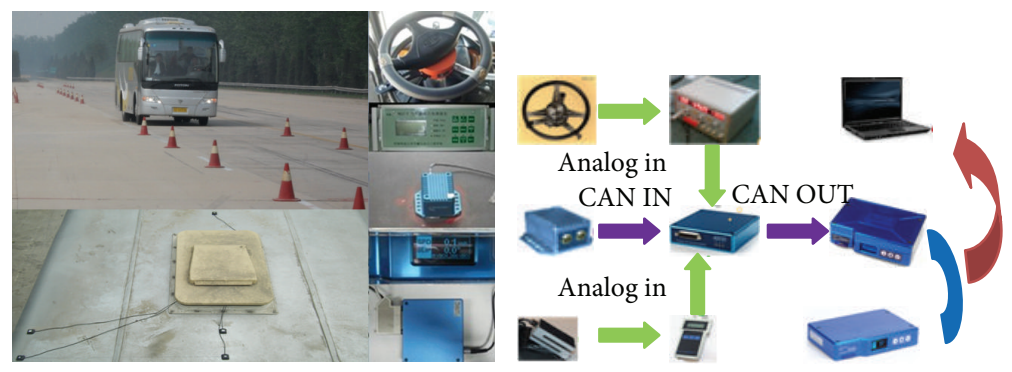

FIGURE 2: Details of vehicle tests and related equipment.

TABLE 1: Curve factors' values of nonlinear tire.

\begin{tabular}{lcccc}
\hline Tire position (number) & $B$ & $C$ & $D$ & $E$ \\
\hline Front wheel (2) & 10.30 & \multirow{2}{*}{1.30} & -11821.63 & -1.05 \\
Rear wheel (4) & 10.51 & & -13437.49 & \\
\hline
\end{tabular}

$$
\begin{gathered}
M_{\psi}=M_{\psi f}+M_{\psi r}, \\
M_{\psi f}=K_{f} \psi_{s}+D_{f} \dot{\psi}_{s}, \\
M_{\psi r}=K_{r} \psi_{s}+D_{r} \dot{\psi}_{s} .
\end{gathered}
$$

2.2. Nonlinear Tire Model. The lateral tire force depending on the side slip angle is represented as the Pacejka magic formula [9]:

$$
F_{y}=D \sin (C \arctan \{B \alpha-E[B \alpha-\arctan (B \alpha)]\}),
$$

where $\alpha$ is the slip angle of tire and $B, C, D$, and $E$ are, respectively, stiffness factor, shape factor, peak factor, and curvature factor. These factors are obtained by fitting. According to the structure of vehicle tire and vertical load, the relevant factor values of tire on normal $\operatorname{road}(\mu=0.7)$ are listed (Table 1) [10].

2.3. Vehicle Dynamic Model Validation. Vehicle test on normal road is to verify the vehicle dynamic model. The details of tests environment and related equipment are showed (Figure 2).
As shown in Figure 2, the integrated test system mainly involves HCZ-1 vehicle handling force and angle measuring instrument, inertial sensors IMU02, vehicle motion parameters measuring instrument by GPS called RLVB20SL, data collection device RLVB-ADC03, and computer. Function of the system is to measure the steering wheel angle input and vehicle motion parameters, such as longitudinal, lateral, and vertical velocity, acceleration, angle, and angular velocity.

The test data is processed by VBOX Tools and Matlab. The position deviations between the actual locations of the test equipment and the vehicle's centroid should be taken into account, so processing the sampling data is necessary and a key step [11].

(1) Inertial Sensors IMU02 Direction Correction. Coordinate system of IMU02 (Figure 3) is corrected by right-hand rule and detail (Table 2).

(2) Vehicle Centroid State Parameters Correction. According to the deviation between the actual locations of the test equipment and the vehicle's centroid, sampling data of RLVB20SL and IMU02 should be processed as follows.

(1) Vehicle Longitudinal Velocity and Acceleration. Consider

$$
\begin{aligned}
& v_{x}=v_{x \mathrm{GPS}}-s_{z 1} \dot{\gamma}_{\mathrm{INS}}, \\
& a_{x}=a_{x \mathrm{INS}}-s_{z 2} \ddot{\gamma}_{\mathrm{INS}},
\end{aligned}
$$


TABLE 2: Coordinate system of IMU02 correction.

\begin{tabular}{lcccccc}
\hline Parameter & Longitudinal & Lateral & Vertical & Yaw & Roll & Pitch \\
\hline Direction correction & + & + & - & - & & + \\
\hline
\end{tabular}

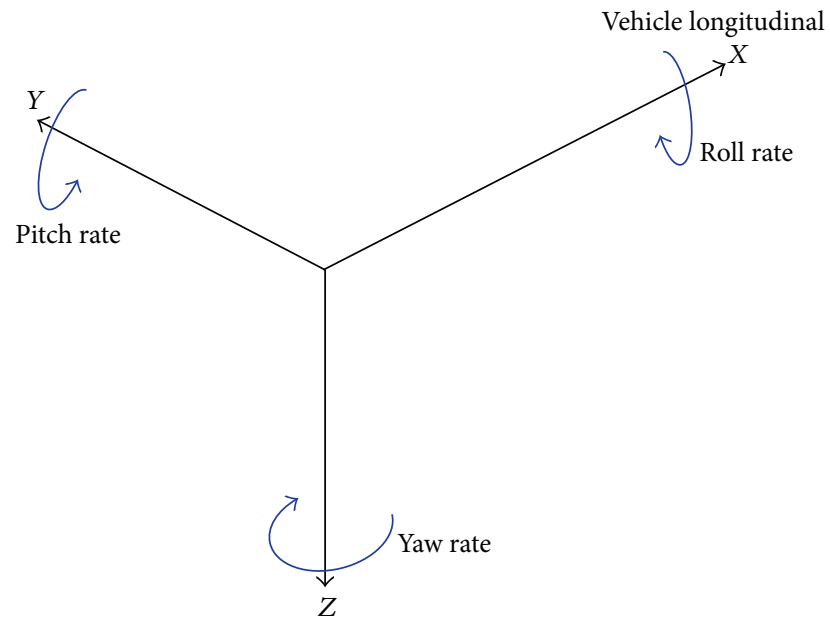

FIGURE 3: IMU02 coordinate.

where $v_{x \mathrm{GPS}}$ is longitudinal velocity tested by RLVB20SL, $a_{x \mathrm{INS}}$ and $\gamma_{\mathrm{INS}}$ are longitudinal acceleration and pitch angle tested by IMU02, $s_{z 1}$ is vertical distance between the main antenna of RLVB20SL and vehicle centroid, and $s_{z 2}$ is vertical distance between the location of IMU02 and vehicle centroid.

(2) Vehicle Lateral Velocity and Acceleration. Consider

$$
\begin{aligned}
& v_{y}=v_{y \mathrm{GPS}}-s_{x 1} \omega_{\mathrm{INS}}+s_{z 1} \dot{\psi}_{\mathrm{INS}}, \\
& a_{y}=a_{y \mathrm{INS}}-s_{x 2} \dot{\omega}_{\mathrm{INS}}+s_{z 2} \ddot{\psi}_{\mathrm{INS}},
\end{aligned}
$$

where $v_{y \mathrm{GPS}}$ is lateral velocity tested by RLVB20SL, $a_{y \mathrm{INS}}$, $\omega_{\text {INS }}$, and $\Psi_{\text {INS }}$ are lateral acceleration, yaw rate, and roll angle tested by IMU02, $s_{x 1}$ is longitudinal distance between the main antenna of RLVB20SL and vehicle centroid, and $s_{x 2}$ is longitudinal distance between the location of IMU02 and vehicle centroid.

As shown in Figure 2, the tested vehicle is driven along the double change race on normal road [12]. The velocity and steering wheel angle operated by driver are showed in Figure 4(a). Then, under the same condition, vehicle lateral acceleration by vehicle tests and Matlab simulation applying the established model is obtained, as shown in Figure 4(b).

Clearly, vehicle's lateral acceleration in Figure 4 shows the good consistency between simulation and vehicle test, and the error is less than $10 \%$. So the reliability of the established nonlinear dynamic model is definitely concluded and could be used for the vehicle stability analysis described in the next section.

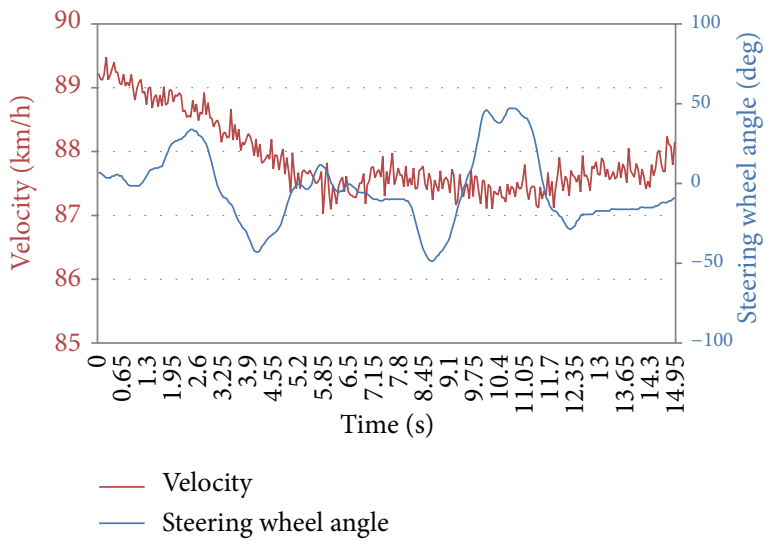

(a) Vehicle's velocity and steering wheel angle input

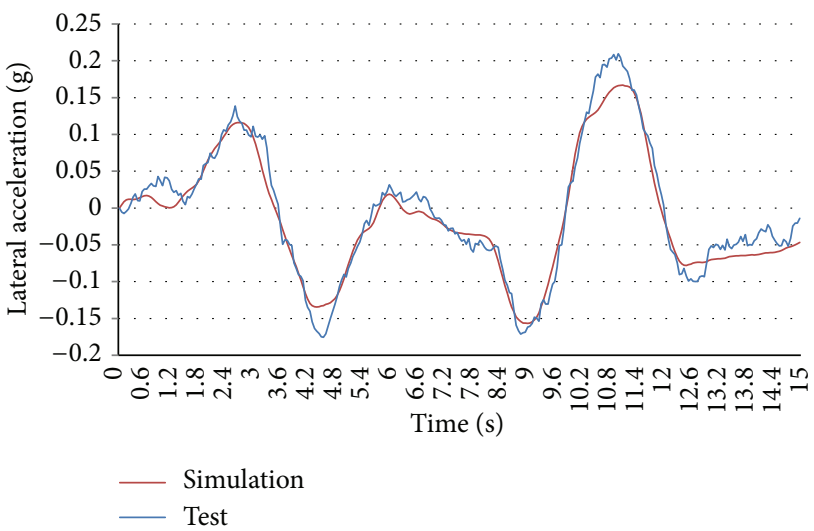

(b) Vehicle's lateral acceleration output

FIgURE 4: Comparing simulation with vehicle test.

\section{Vehicle Steering Stability Analysis}

Suppose $\left(\mathbf{X}^{e}, \delta_{f}^{e}\right)$ and $\mathbf{X}^{e}=\left(v_{y}^{e}, \omega^{e}, \dot{\psi}_{s}^{e}, \psi_{s}^{e}\right)$ are one of the balance points of vehicle system. Then the nonlinear formula $\dot{\mathbf{x}}=f(\mathbf{x})$ could be described as

$$
\dot{\mathbf{x}}=A \mathbf{x}+F\left(\mathbf{x}, \delta_{f}\right),
$$

where $A$ is Jacobian matrix located on the point of $\left(\mathbf{X}^{e}, \delta_{f}^{e}\right)$.

If $\delta_{f} \neq 0$, the Jacobian matrix $A$ is expressed as

$$
\begin{aligned}
A & =\left.D_{x} f\left(\mathbf{X}, \delta_{f}\right)\right|_{\left(v_{y}^{e}, \omega^{e}, \dot{\psi}_{s}^{e}, \psi_{s}^{e}\right)} \\
& =\left[\begin{array}{llll}
A_{11} & A_{12} & A_{13} & A_{14} \\
A_{21} & A_{22} & A_{23} & A_{24} \\
A_{31} & A_{32} & A_{33} & A_{34} \\
A_{41} & A_{42} & A_{43} & A_{44}
\end{array}\right] .
\end{aligned}
$$




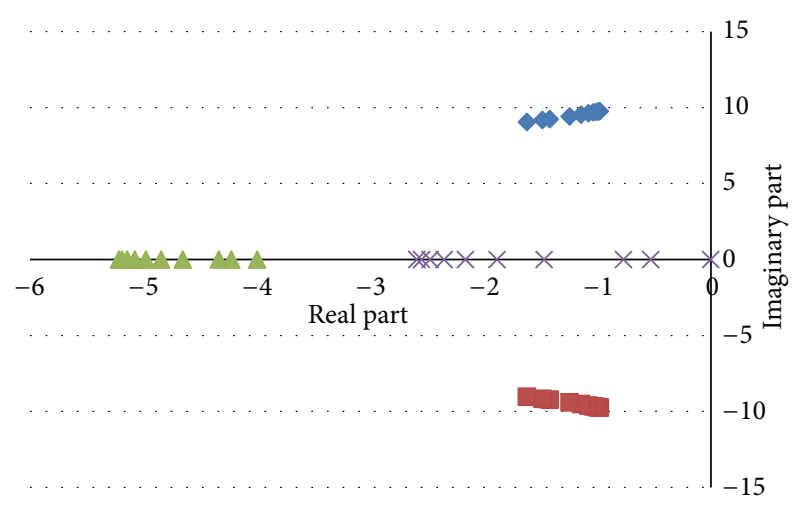

Characteristic root 1 Characteristic root 3

Characteristic root $2 \times$ Characteristic root 4

FIGURE 5: Characteristic roots of Jacobian matrix.

Characteristic equation of Jacobian matrix $A$ is as follows:

$$
\lambda^{4}+c_{1} \lambda^{3}+c_{2} \lambda^{2}+c_{3} \lambda+c_{4}=0
$$

Hurwitz principle shows the characteristic of the equation $[13,14]$

$$
\begin{gathered}
\Delta_{1}=c_{1}>0, \\
\Delta_{2}=c_{1} c_{2}-c_{3}>0, \\
\Delta_{3}=c_{3}\left(c_{1} c_{2}-c_{3}\right)>0, \\
\Delta_{4}=c_{3} c_{4}\left(c_{1} c_{2}-c_{3}\right)>0 .
\end{gathered}
$$

According to Hurwitz principle, if the $c_{1} \sim c_{4}$ satisfy (9), the characteristic roots are all negative. If the $c_{1} \sim c_{4}$ do not satisfy (9) and $c_{4} \neq 0$, positive characteristic root will exist, which predict the unbalance of vehicle system. When the vehicle speed is $20 \mathrm{~m} / \mathrm{s}$, the front wheel steering angle is in $[0,0.042] \mathrm{rad}$ and interval $0.005 \mathrm{rad}$, Jacobian matrix characteristic roots with the changes of front wheel steering angle shown in Figure 5.

Note that the arrow indicates the change direction of the Jacobian matrix characteristic roots in Figure 5. It is shown that the characteristic roots are all negative when the front wheel steering angle is small, but they gradually approach 0 as the angle increases and then one of the characteristic roots is 0 if the angle reaches a certain value called critical steering angle. The results given by (a) and (b) in Figure 4 represent characteristic root which is a good way to judge the stability and obtain the critical steering angle and state parameters of vehicle system.

\section{Stability Regions Estimation}

4.1. Energy Function Establishing. Assume a quadratic energy function $V(x)$ as follows [15]:

$$
\begin{gathered}
V(x)=x^{T} P x, \\
\dot{V}(x)=\dot{x}^{T} P x+x^{T} P \dot{x},
\end{gathered}
$$

where $x^{T}=\left[v_{y}, \omega, \dot{\psi}_{s}, \psi_{s}\right]$ and " $P$ " is a positive definite symmetric matrix:

$$
P=\left[\begin{array}{llll}
p_{11} & p_{12} & p_{13} & p_{14} \\
p_{12} & p_{22} & p_{23} & p_{24} \\
p_{13} & p_{23} & p_{33} & p_{34} \\
p_{14} & p_{24} & p_{34} & p_{44}
\end{array}\right]
$$

The matrix " $P$ " is obtained from the next equation:

$$
A^{T} P+P A=-I
$$

where " $I$ " is unit matrix and " $A$ " is the system's Jacobian matrix in origin: $A=\left.(\partial f(x) / \partial x)\right|_{x=0}$.

Fact is obvious that the Jacobian matrix is a function of vehicle velocity, so, based on the reference equations, the matrices " $A$ " and " $P$ " in different driving velocities and the corresponding energy functions $V(x)$ and $\dot{V}(x)$ could be obtained. The matrices " $A$ " and " $P$ " when the vehicle's velocity is $20 \mathrm{~m} / \mathrm{s}$ are listed:

$$
\begin{aligned}
A & =\left[\begin{array}{cccc}
3.725 & -2.007 e 1 & -7.956 & -1.384 e 2 \\
-6.640 e-2 & -7.503 & -9.950 e-2 & -1.732 \\
1.031 e 1 & 4.140 e-1 & -1.048 e 1 & -1.823 e 2 \\
0 & 0 & 1 & 0
\end{array}\right], \\
P & =\left[\begin{array}{cccc}
2.466 e-1 & -2.999 e-1 & -1.395 e-1 & -2.0534 \\
-2.999 e-1 & 8.899 e-1 & 3.815 e-1 & 1.638 \\
-1.395 e-1 & 3.815 e-1 & 2.976 e-1 & 1.5465 \\
-2.0534 & 1.638 & 1.5465 & 3.563 e 1
\end{array}\right] .
\end{aligned}
$$

4.2. Boundary Conditions of Stability Region. Generally speaking, traditional principle of the boundary conditions of driving stability region depends on energy attenuation called Lyapunov's second theorem. Actually, energy attenuation is not enough to calculate the stability region accurately because vehicle is a regular system when it is stable and the space estimated by the energy attenuation characteristic is unreasonably expanded. So the critical vehicle state based on the vehicle system stability analysis should be introduced to determine the vehicle stability region.

Boundary conditions mainly lie in two aspects: first, according to Lyapunov's second theorem, $\dot{V}(x) \leq 0$ is the sufficient condition for system asymptotically stable [16]. Second, steady critical steering angle of front wheel and the corresponding related vehicle state parameters could be obtained by Hurwitz principle, as listed in Table 3 .

Consequently, the boundary conditions of the vehicle driving stability regions could be described as follows:

$$
\begin{gathered}
\dot{V}(x)=0, \\
V(x)=V_{c} .
\end{gathered}
$$

4.3. Vehicle Driving Stability Regions Estimation. According to the proposed boundary conditions of the vehicle stability regions, Figure 7 vividly describes the critical surfaces in terms of the boundary conditions. 
TABLE 3: Critical values of $V(x)$.

\begin{tabular}{lccccr}
\hline$u(\mathrm{~m} / \mathrm{s})$ & $\delta_{c}(\mathrm{rad})$ & $\beta(\mathrm{rad})$ & \multicolumn{2}{c}{ Stable equilibrium points of state variables } & $\psi_{s}(\mathrm{rad})$ \\
\hline 20 & 0.042 & -0.067 & $\omega(\mathrm{rad} / \mathrm{s})$ & $\dot{\psi}_{s}(\mathrm{rad} / \mathrm{s})$ & 0.240 \\
\hline
\end{tabular}

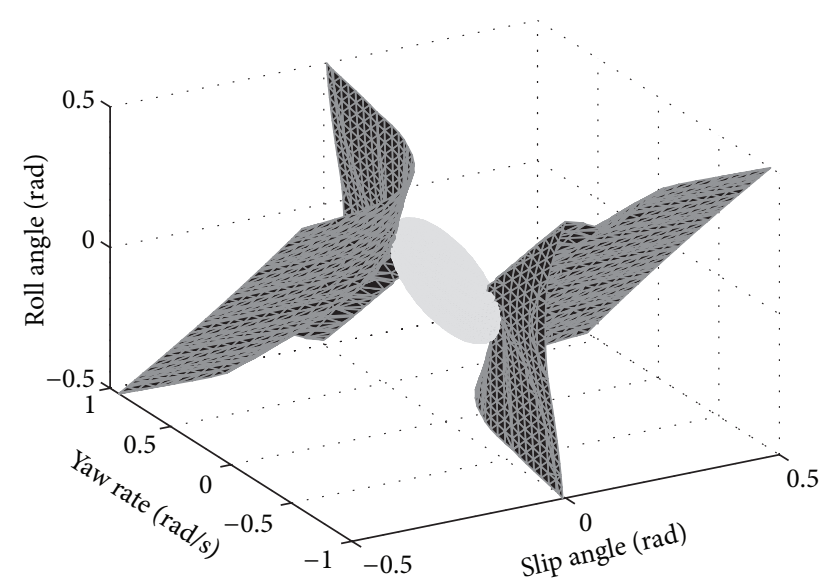

$\dot{V}(x)=0$

$V(x)=0.15$

FIgURE 6: Critical surfaces in terms of the boundary conditions.

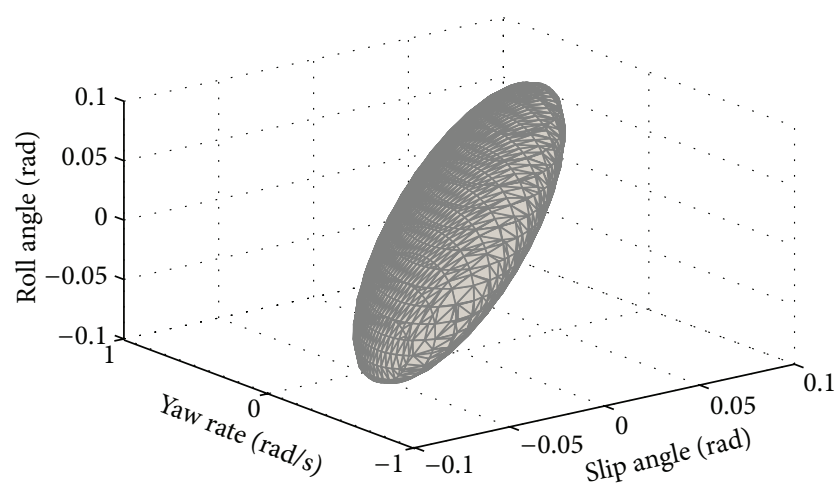

FIGURE 7: Stability region defined by $V(x)=0.15$ in $20 \mathrm{~m} / \mathrm{s}$.

As shown in Figure 6, for $\dot{V}(x)=0$ and any value of vector $x, \dot{V}(x)$ is a complexity function of vector $x$ due to the Pacejka tire magic formula, so irregular symmetrical ridge surfaces are formed. Besides, the $V(x)=V_{c}$, as quadratic functions of vector $x$, are described by symmetrical ellipsoids.

Obviously, the space region determined by $\dot{V}(x)=0$ driving stability regions is obtained by the intersection of $\dot{V}(x)=0$ and $V(x)=V_{c}$. So the stability regions are, respectively, showed in Figure 7.

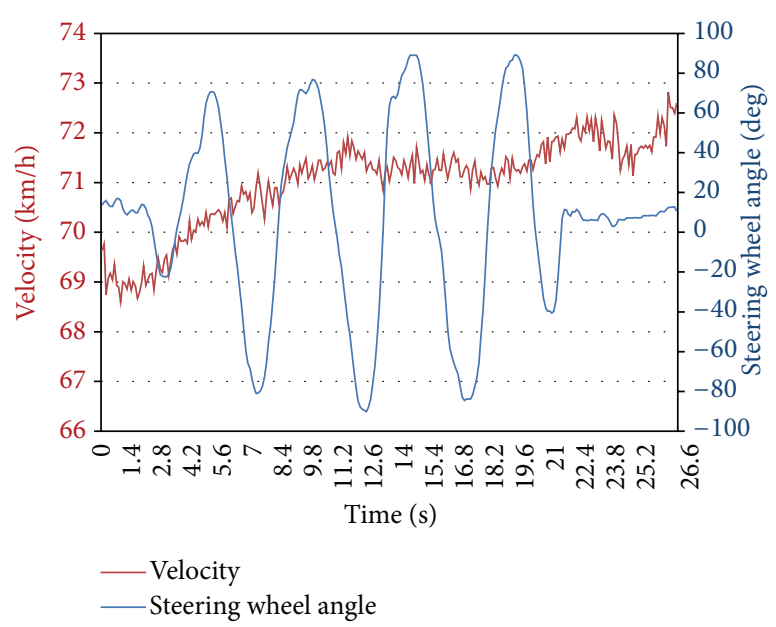

FIgURE 8: Test velocity and steering angle.

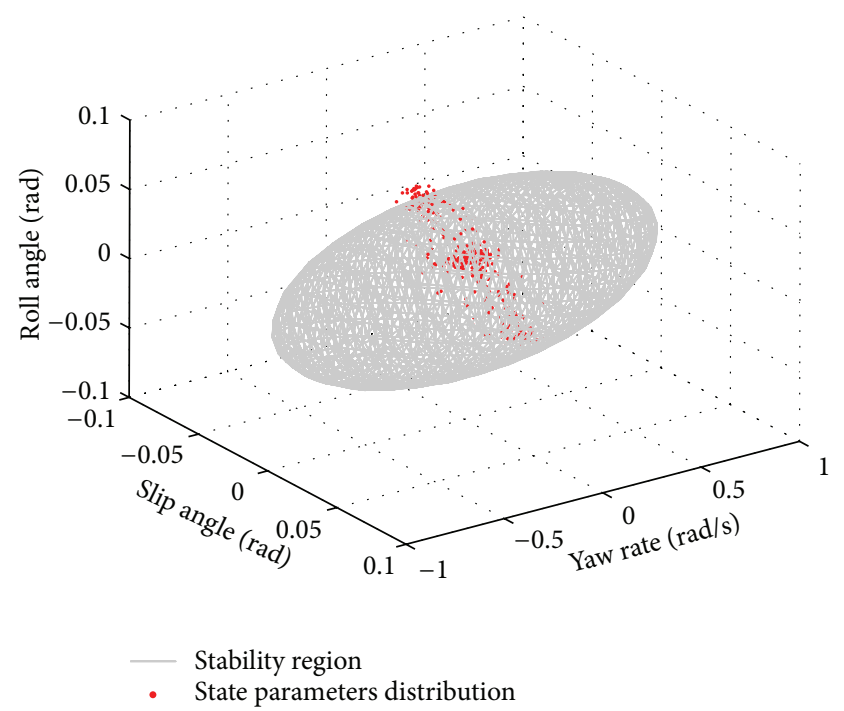

FIGURE 9: Comparing the stability region to vehicle tests results.

\section{Stability Region Validation}

Appling the integrated test system, vehicle snake shape dynamic behavior is tested. Details of the vehicle's velocity and steering wheel angles with time are showed in Figure 8.

Figure 9 shows the corresponding driving stability region and the obtained distribution of slip angle, yaw rate, and roll angle. Besides, for sake of well comparing the test results with the calculated driving stability region, details of yaw rate and roll angle phase plane are illustrated in Figure 11.

As shown in Figure 9, it is clear that when the vehicle is stable, the distributions of the relevant state variables 


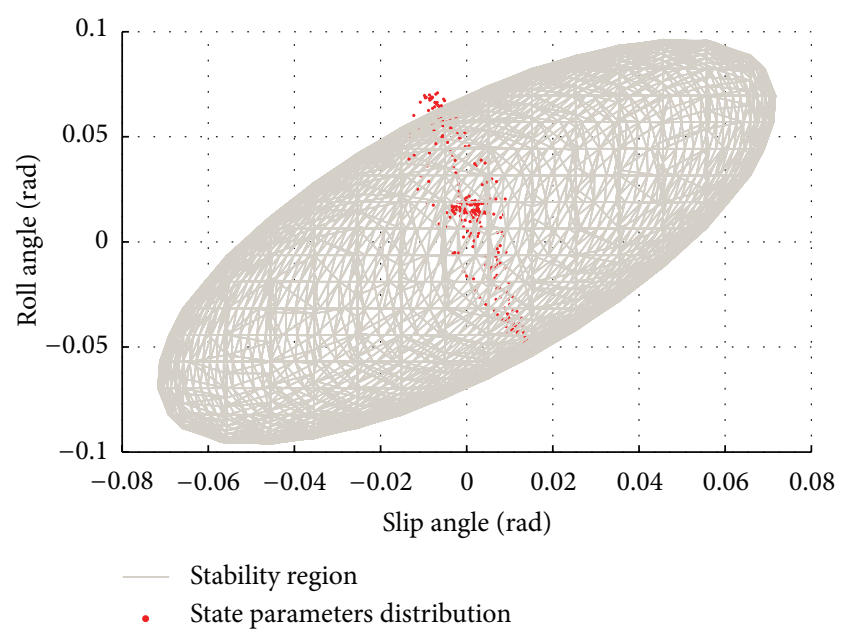

FIGURE 10: Details of vehicle's dynamic parameters' distribution.

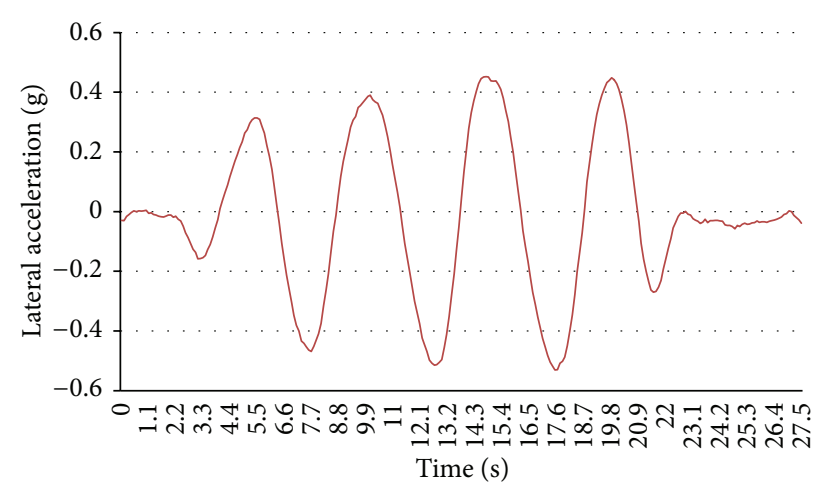

FIgURE 11: Vehicle's lateral acceleration with time.

of vehicle are both in the stability region. However, as the steering wheel angle increases and vehicle system has approached its critical state, trend from stability to instability is obvious. When the steering wheel angle reaches the critical value, the distribution of the state variables while skidding is outside the stability region. Details are showed in Figure 10.

Figure 11 represents the lateral acceleration of the vehicle and the maximum value of the acceleration is up to $0.5 \mathrm{~g}$ which has beyond the critical state. Actually, there is slip phenomenon during the test.

The contrast results indicate that the stability region of the vehicle's stability has good consistency with the test results. Therefore, the proposed driving stability region is reliable for judging and evaluating the stability of vehicle.

\section{Conclusion}

(1) 3DOF vehicle dynamic model with nonlinear tire formulas is deduced and simulated by Matlab. Applying advanced GPS and inertial measurement technology, detecting equipment and data process for vehicle handing stability is integrated and developed, and vehicle in double lane change conditions is tested and the established vehicle model is verified.
The reliability of the established nonlinear dynamic model is clearly concluded and could be used for the vehicle stability analysis described in the next section.

(2) Law of vehicle system's Jacobian matrix characteristic roots changing with increased front wheel steering angles are fundamentally revealed based on Hurwitz principle. Consequences of the characteristics introduce a good method for defining the boundary of stability region.

(3) The driving stability region of vehicle verified by vehicle snake shape test provides an effective reference for stability evaluation. The consequences of the vehicle space stability region introduce the new insight into the vehicle performance of stability.

\section{Conflict of Interests}

The authors declare that there is no conflict of interests regarding the publication of this paper.

\section{Acknowledgments}

The authors received sponsorship from the Key Project of the National Eleventh-Five Year (no. 2009BAG13A04) and the National High Technology Research and Development Program 863 (no. 2009AA11Z201).

\section{References}

[1] T. D. Gillespic, Fundamentals of Vehicle Dynamics, Society of Automotive Engineers, Warrendale, Pa, USA, 2000.

[2] R. N. Jazar, Vehicle Dynamics: Theory and Application, Springer, Berlin, Germany, 2009.

[3] G. S. Inagaki, L. Kushiro, and M. Yamamoto, "Analysis on vehicle stability in critical cornering using phase-plane method," SAE Paper 9438411, 1994.

[4] J. Samsundar and J. C. Huston, "Estimating lateral stability region of a nonlinear 2 degree-of-freedom vehicle," SAE paper 981172, 1998.

[5] Y. Yasui, K. Tozu, N. Hattori, and M. Sugisawa, "Improvement of vehicle directional stability for transient steering maneuvers using active brake control," SAE Paper 960485, 1996.

[6] A. Stotsky and X. Hu, "Stability analysis of robustly decoupled car steering system with nonlinear tire model," in Proceedings of the 36th IEEE Conference on Decision and Control, pp. 47504755, December 1997.

[7] Y. E. Ko and J. M. Lee, "Estimation of the stability region of a vehicle in plane motion using a topological approach," International Journal of Vehicle Design, vol. 30, no. 3, pp. 181192, 2002.

[8] S. Shen, J. Wang, P. Shi, and G. Premier, "Nonlinear dynamics and stability analysis of vehicle plane motions," Vehicle System Dynamics, vol. 45, no. 1, pp. 15-35, 2007.

[9] B. Pacejka Hance, Tyre and Vehicle Dynamics, Society of Automotive Engineers, Warrendale, Pa, USA, 2002.

[10] G. Tian, M. Zhou, and J. Chu, "A chance constrained programming approach to determine optimal disassembly sequence," IEEE Transactions on Automation Science and Engineering, vol. 10, no. 4, pp. 1004-1013, 2013. 
[11] G. Tian, M. Zhou, J. Chu, and Y. Liu, "Probability evaluation models of product disassembly cost subject to random removal time and different removal labor cost," IEEE Transactions on Automation Science and Engineering, vol. 9, no. 2, pp. 288-295, 2012.

[12] BS ISO 3888-1:1999, "Passenger cars. Test track for a severe lanechange manoeuvre. Double-lane change".

[13] H. True, "On the theory of nonlinear dynamics and its applications in vehicle systems dynamics," Vehicle System Dynamics, vol. 31, no. 5-6, pp. 393-421, 1999.

[14] Z. Liu and G. Payre, "Global bifurcation analysis of a nonlinear road vehicle system," Journal of Computational and Nonlinear Dynamics, vol. 2, no. 4, pp. 308-315, 2007.

[15] Z. GAjic, Lyapunov Matrix Equation in System Stability, Academic Press, London, UK, 1995.

[16] V. Nguyen, G. Schultz, and B. Balachandran, "Lateral load transfer effects on bifurcation behavior of four-wheel vehicle system," Journal of Computational and Nonlinear Dynamics, vol. 4, no. 4, pp. 1-12, 2009. 


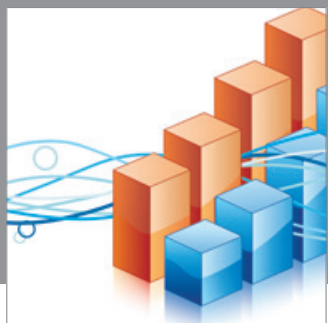

Advances in

Operations Research

mansans

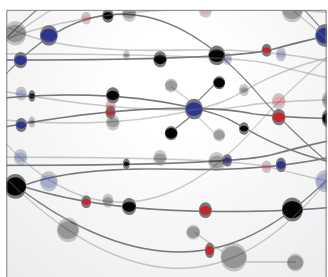

The Scientific World Journal
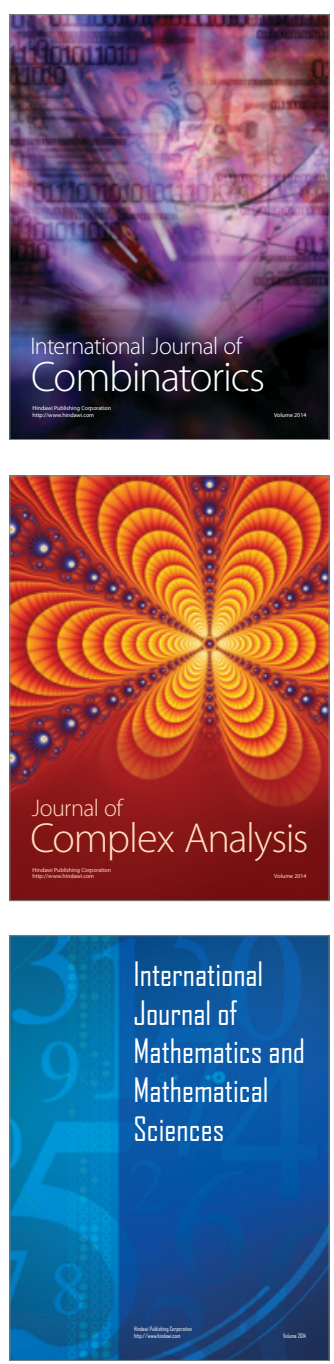
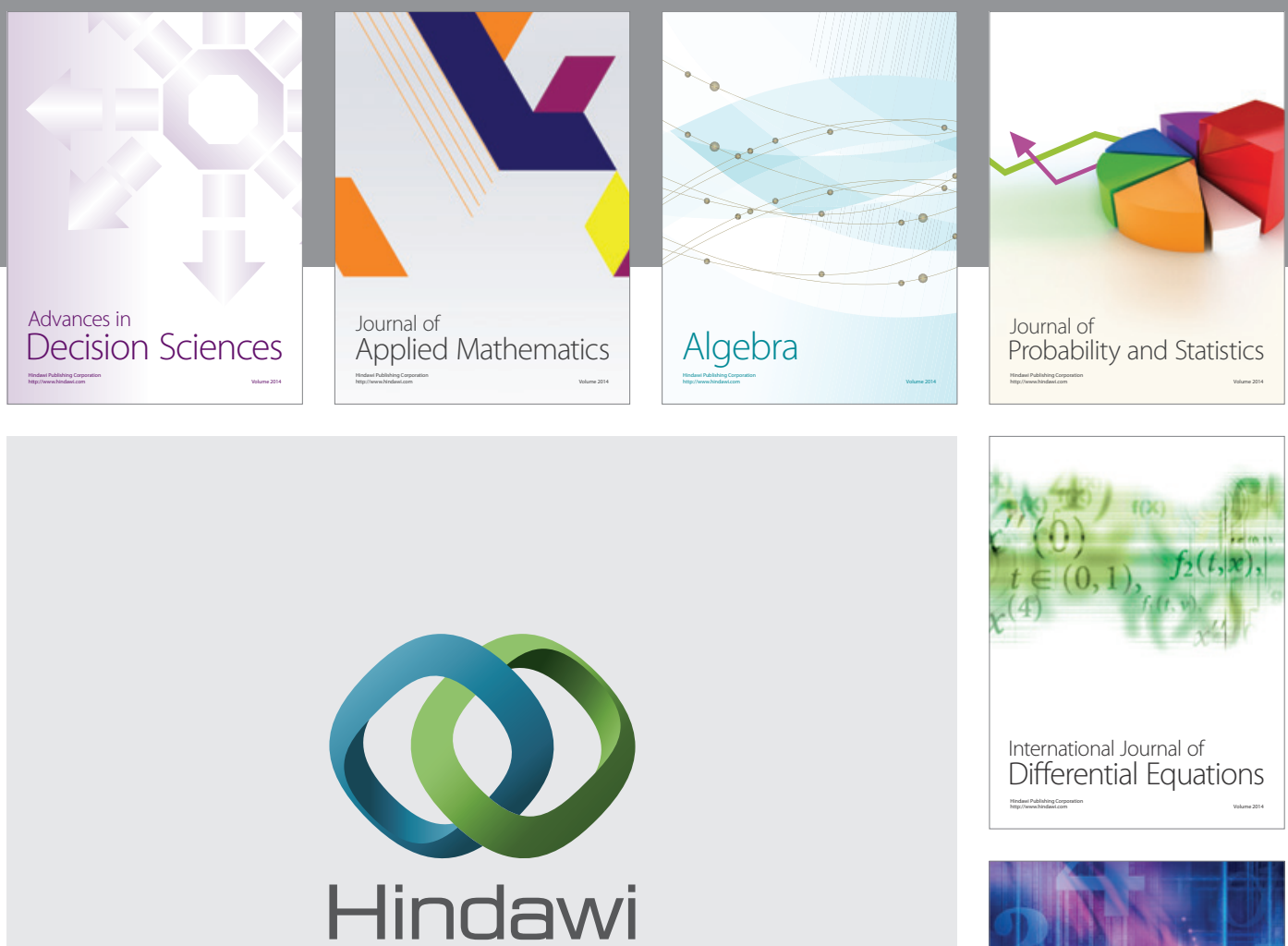

Submit your manuscripts at http://www.hindawi.com
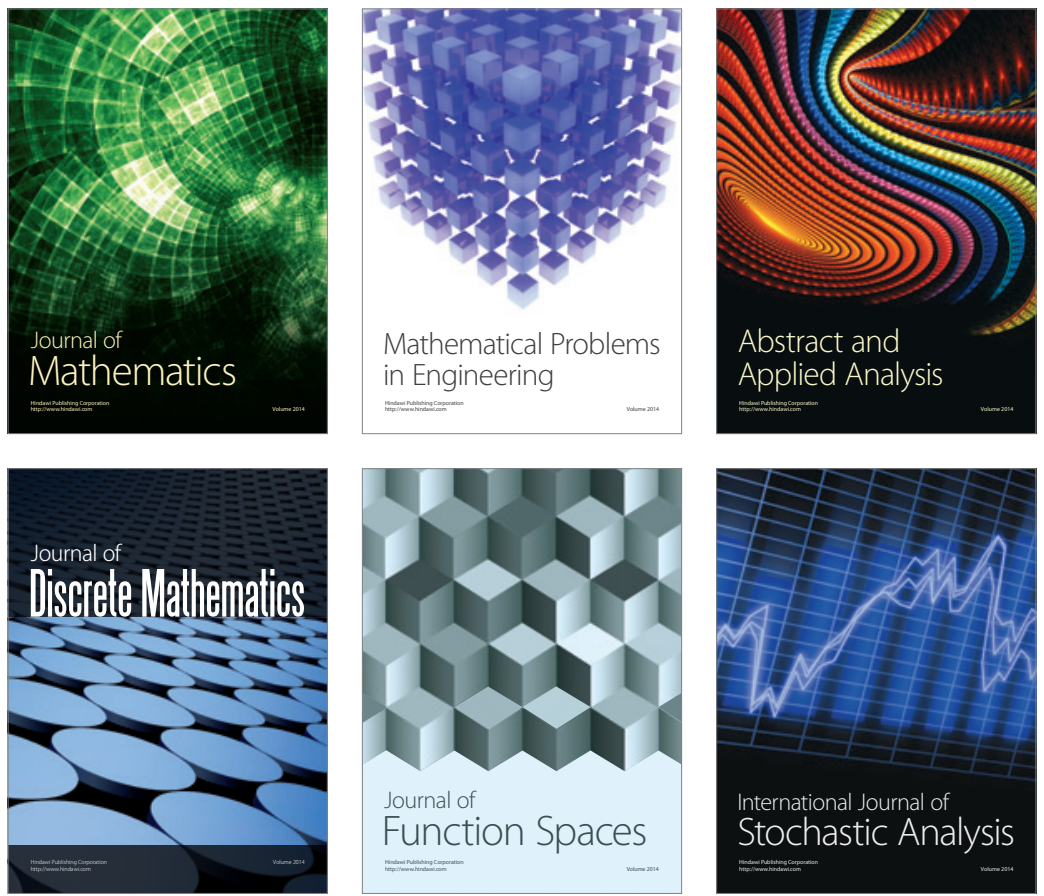

Journal of

Function Spaces

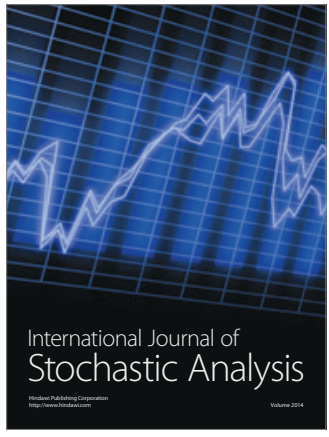

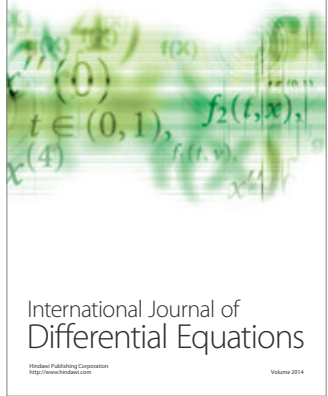
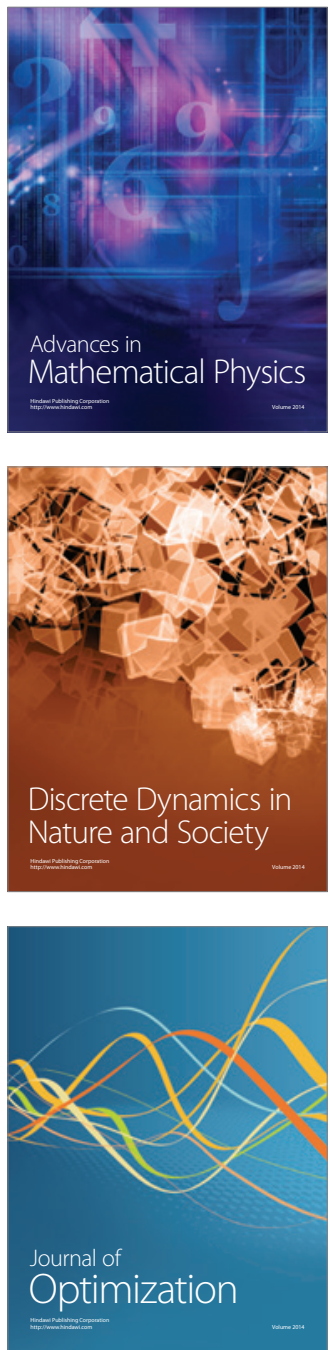